\title{
Rectal Cancer Metastasis to the Anal Verge: An Unusual Case Presentation and Review of the Literature
}

\author{
Garrett GRJ Johnson (D) ${ }^{1,2}$ \\ Benson Yip' \\ Ramzi M Helewa' \\ Farhana Shariff' \\ Eric Hyun' \\ 'Department of Surgery, Section of \\ General Surgery, University of Manitoba, \\ Winnipeg, Manitoba, Canada; ${ }^{2}$ Clinician \\ Investigator Program, University of \\ Manitoba, Winnipeg, Manitoba, Canada
}

Correspondence: Eric Hyun

Email ehyun@sbgh.mb.ca
Background: Anal metastasis of colorectal adenocarcinoma is very rare, represented by only a handful of case reports in the literature. Previously, reports of metastasis to this region had occurred following a history of anorectal disease, such as anal fistulae. Antecedent trauma to the area from hemorrhoidectomy, fissures, or perineal retractor injury have also been implicated.

Case Presentation: Herein we report the case of 69-year-old man without any history of anal disease presenting with a metachronous metastasis of a colorectal-type adenocarcinoma to the anal verge. He was previously treated for T1N0 rectal adenocarcinoma at the rectosigmoid junction with a low anterior resection 5 years prior, then had a T3N0 local recurrence at the colorectal anastomosis treated with neoadjuvant chemoradiation, and eventually a Hartmann's procedure 4 years later. Subsequently, on surveillance flexible sigmoidoscopy, a new tumor was identified on the perianal skin extending from the anal verge. Histopathology demonstrated colorectal-type adenocarcinoma. Flexible endoscopy identified no other residual or recurrent disease in the colon or rectal stump. The patient was treated with wide local excision and advancement flap reconstruction.

Conclusion: Isolated metastasis to the anus is an extremely rare occurrence for colorectal adenocarcinoma. There exists little evidence to inform management. One option is to treat like a locally recurrent rectal cancer with aggressive tri-modality management consisting of chemoradiation, abdominal perineal resection, and adjuvant chemotherapy. In the absence of metastatic disease, local resection and close surveillance remain an option. As always, patient factors should guide management.

Keywords: colorectal cancer, anal metastasis, rectal cancer, anal adenocarcinoma

\section{Introduction}

Colorectal cancer is the fourth most common malignancy globally. ${ }^{1}$ Up to $22 \%$ of patients in the United States with this disease present with metastases. ${ }^{2}$ However, anal metastases are exceedingly rare. Some have estimated that metastasis to this location occurs in less than $1 \%$ of patients with this disease. ${ }^{3,4}$ In nearly all previously reported cases in the literature, anal metastasis was associated with a history of antecedent anal disease such as pre-existing perianal fistulae or fissures. $^{3-13}$ Implantation into the surgical site following hemorrhoidectomy has also been noted on multiple occasions. ${ }^{14-20}$ Tumor implantation from trauma during rectal cancer operative procedures has also been described. ${ }^{21-23}$ To our knowledge, isolated anal metastasis to intact perianal surface tissue in the absence of epithelial 
injury has only been described once before in the literature. ${ }^{24}$ Herein, we present the case of a 69 -year-old man with a colorectal cancer originally at the rectosigmoid junction, presenting with a recurrence at the anal verge 5 years later, with no history of prior anal disease or trauma.

\section{Case Presentation}

The patient originally presented at age 64 to a community hospital for an asymptomatic screening colonoscopy. He had a personal history of two small adenomatous colon polyps removed in a past endoscopy but no personal or family history of any cancers at that time. That colonoscopy identified a polyp of $3 \mathrm{~cm}$ diameter at $20 \mathrm{~cm}$ from the anal verge. This was tattooed by the endoscopist and removed via an endoscopic submucosal dissection. Unfortunately, on histopathology this was diagnosed as a low-grade colon adenocarcinoma with a microscopically positive margin. His blood CEA level was $1.1 \mu \mathrm{g} / \mathrm{L}$, and his staging CT scan of the chest, abdomen and pelvis demonstrated no signs of metastatic disease, so he subsequently underwent low anterior resection with primary anastomosis. The specimen contained 30 normal regional lymph nodes without evidence of residual or metastatic disease. He was staged as T1N0M0 colon adenocarcinoma at that time, and followed routine surveillance with his surgeon. His post-operative course was complicated by an anastomotic stricture, which was effectively managed with serial endoscopic dilatations by his surgeon.

Two years later, he developed some new onset rectal bleeding. His CEA rose to $7.4 \mu \mathrm{g} / \mathrm{L}$, and he underwent flexible sigmoidoscopy which identified a friable mass at the colorectal anastomosis, $8 \mathrm{~cm}$ from the anal verge. This was biopsied and demonstrated recurrent rectal cancer (MSI stable and MLH1/PMS2/MSH2/MSH6 intact). Staging CT scans and rectal MRI exam demonstrated a locally advanced lesion with spread into the peri-rectal fat, but no nodal metastasis, and he was referred to our tertiary care centre for neoadjuvant therapy and a surgical consultation. Over the subsequent months, he received long course neoadjuvant chemoradiation (capecitabine and 50.4 Gy in 28 fractions). At 6 weeks after completing neoadjuvant chemoradiation, his CEA had decreased to $2.3 \mu \mathrm{g} / \mathrm{L}$, and flexible sigmoidoscopy and CT scans demonstrated no clinical evidence of residual disease. The patient was offered oncologic resection at our hospital; however, he had developed significant deconditioning and fatigue which he attributed to his neoadjuvant therapy, and felt that he could not cope with another operation at that time. Against our recommendations, he declined surgery in favor of a "watch and wait" approach.

He was closely monitored with CEA, CT scans and flexible sigmoidoscopy. Two years later, his CEA suddenly rose to $9.0 \mu \mathrm{g} / \mathrm{L}$, and another local recurrence was identified on endoscopy, in the same location at the rectal anastomosis. CT scans demonstrated no signs of distant metastatic disease. This time, he agreed to a repeat low anterior resection, and was brought to the operating room. Intraoperatively, he was found to have significant visceral adiposity, a very narrow pelvis and significant fibrosis from his prior surgery and radiation. A primary anastomosis was deemed not practicable, and he underwent a low Hartmann's procedure with an end colostomy. His postoperative histopathology demonstrated a T3 tumor with extension into the peri-rectal fat, but no nodal metastases and negative margins. Postoperatively, he developed a small dehiscence of his rectal stump, which was managed by a brief hospitalization with intravenous antibiotics. He was referred for adjuvant chemotherapy, which he again declined, citing the significant side effects he experienced from his neoadjuvant chemoradiation.

Six months later, he underwent surveillance flexible sigmoidoscopy of the rectal stump by his surgeon. At this time, there was some granulation tissue at the staple line, and a new large, firm lesion, similar in appearance to an acrochordon, at the anal verge (Figure 1). Both sites were biopsied. The rectal stump histopathology showed benign granulation tissue, whereas the anal lesion was rectal type adenocarcinoma. This time, his CEA remained low at $1.7 \mu \mathrm{g} / \mathrm{L}$. The patient was otherwise asymptomatic. He had no inguinal lymphadenopathy on physical exam, and CT scan of the chest, abdomen and pelvis showed no signs of distant metastatic disease. Following multidisciplinary discussion, the patient was considered for abdominal perineal resection (APR) and chemotherapy for management of what was presumed to be another recurrence of his rectal cancer. Consideration was given to the patient's body habitus, significant pelvic fibrosis, and mechanical difficulties encountered during his prior operation. The risks of major oncologic resection were felt to be significant, therefore the patient opted for a more minimalist approach, with local resection and V-Y flap reconstruction, and no chemotherapy. Post-operative pathology demonstrated a rectal-type adenocarcinoma, consistent with his past tumors. The cancer was invading just to the submucosa, and the surgical margins were widely clear. 


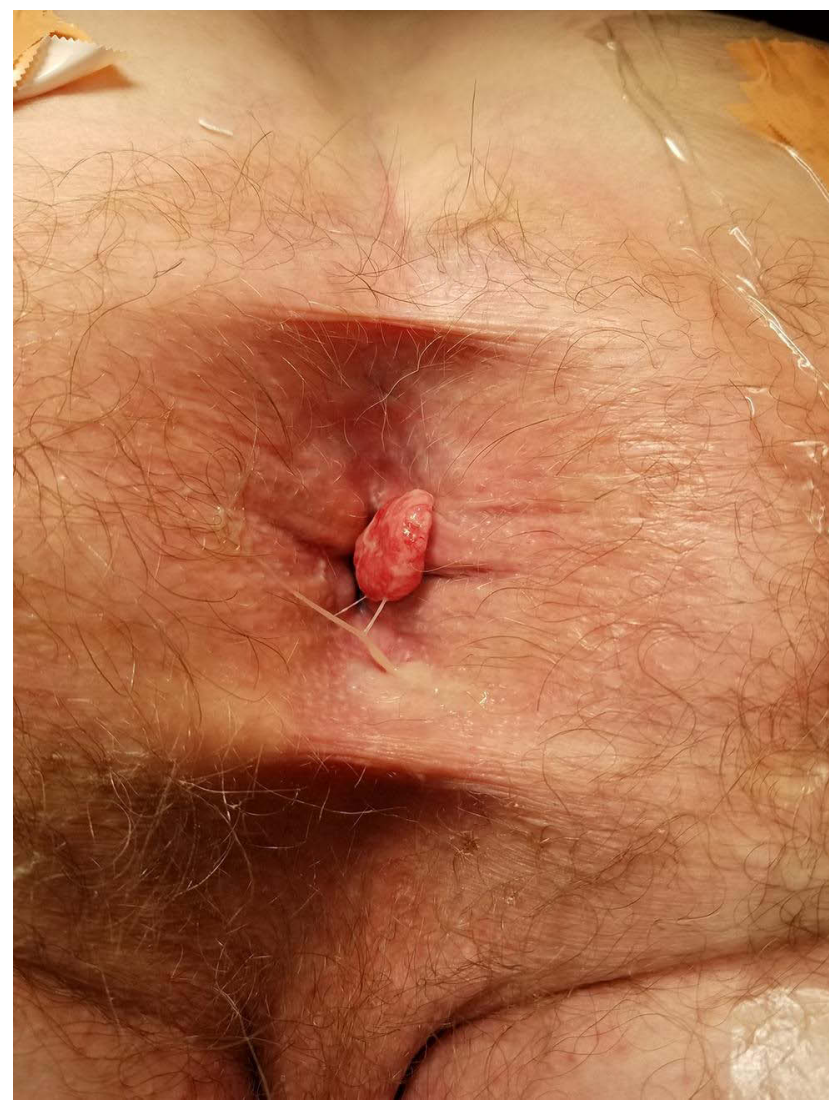

Figure I Preoperative image of the adenocarcinoma metastasis at the anal verge.

Post-operatively, he had a small dehiscence in the suture line of his reconstruction, which healed without any sequelae or intervention (Figure 2). Six months after surgery he continues to do well, without any signs of recurrence.

\section{Discussion}

This case report describes a unique presentation of a relatively common disease. While colorectal cancer metastasis to the anus itself is a rare finding, its presentation without any antecedent trauma or perianal disease has only been described once in the literature. ${ }^{24}$ The current case occurring in an irradiated and re-operative field following multiple local recurrences of a rectosigmoid cancer presents an added therapeutic challenge. Given the paucity of previous reports describing this issue, no guidelines exist to help direct management. Instead, this case report may help us to advance our understanding of this disease, and guide future options for treatment and prevention.

In the above case, the anal metastasis was managed with a local resection of the tumor. This treatment is consistent with that performed in some past case reports of isolated anal metastases, ${ }^{4,21-23}$ which appears to have

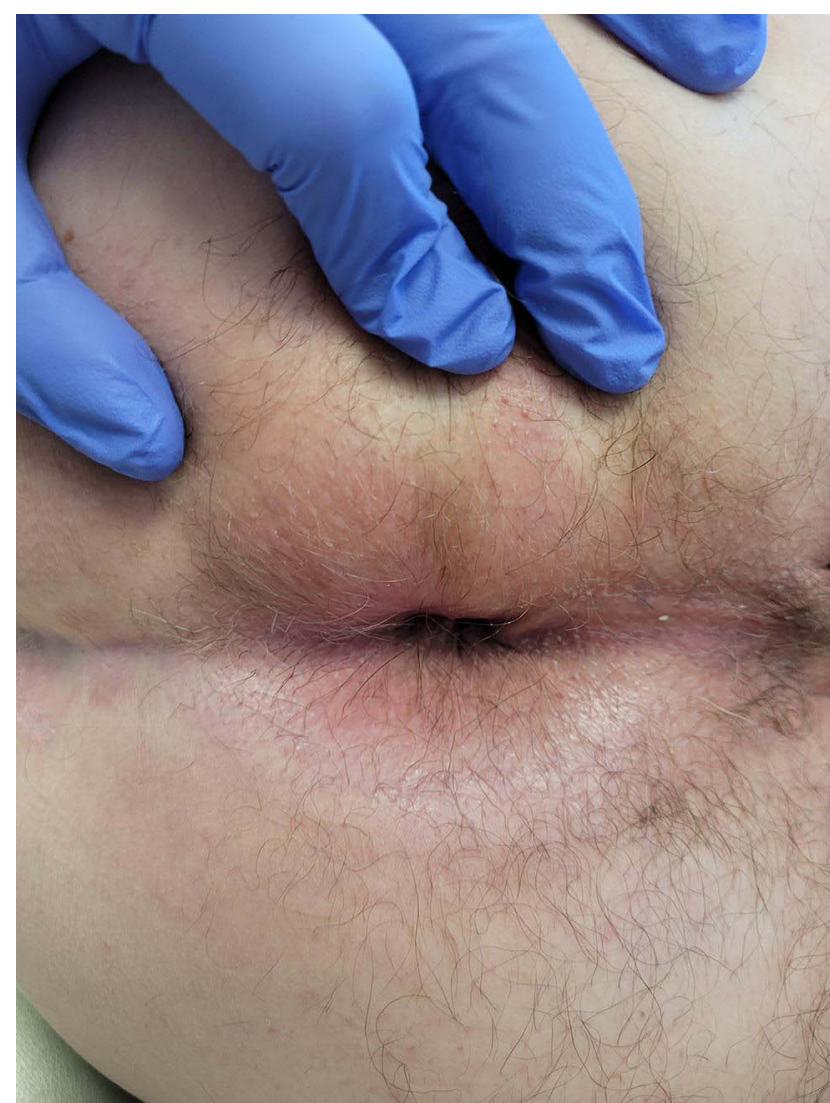

Figure 2 Healed $V-Y$ reconstruction flap on anus at 6 month post-operative visit.

led to long term disease remission in at least one instance. ${ }^{19}$ Other cases have been managed with APR. ${ }^{6,13,15,18,20}$ In the latter cases, patients often presented synchronously with their primary cancer in the rectum, thus necessitating excision of the mesorectum. In the present case, the patient's mesorectal plane had already been violated during his two prior resections. Furthermore, he had significant adiposity, a narrow pelvis, and substantial scarring from his chemoradiation. These are all factors that are described to increase the difficulty of pelvic dissection. ${ }^{25}$ Extrapolating literature from local rectal cancer recurrence suggests that the most important factor for predicting cancer-specific survival in patients undergoing surgery for recurrent rectal cancer was an R0 resection. $^{26,27}$ Therefore, a joint decision between the patient and his providers was made to pursue wide local resection only. Given the negative margins on this specimen, this resection was determined to be adequate.

The decision whether to offer adjuvant chemotherapy in this case was another challenging factor. The patient had not previously received chemotherapy during his prior recurrence due to personal preference. There are no 
prospective trials examining the efficacy of adjuvant chemotherapy following resection of a locally recurrent rectal cancer. The National Comprehensive Cancer Network (NCCN) guidelines suggest cisplatin-based chemotherapy (eg, FOLFOX, CAPEOX) as part of the management algorithm for resectable isolated pelvic recurrence. ${ }^{28}$ Although evidence in support of that recommendation is limited. Retrospective series exist examining the use of adjuvant chemotherapy as a component of multimodality therapy for locally recurrent rectal cancer, however, they do not demonstrate an association between administration of adjuvant chemotherapy and more favorable long-term oncologic outcomes. ${ }^{27,29-31}$ Regardless, applicability of those studies to the current presentation is tenuous. Most cancers included in those analyses had recurred at the anastomotic site or in the pelvis, rather than at the anus. An alternative perspective would be to treat this anal recurrence as a resectable distant metachronous metastasis, rather than a local recurrence. Again, NCCN guidelines suggest the provision of adjuvant chemotherapy for resectable stage IV rectal cancer patients who had not previously received chemotherapy. ${ }^{28}$ However, the evidence of benefit for this treatment is indeterminate, and is extrapolated primarily from colorectal liver metastases. The largest meta-analysis ( $n=1896$ patients) of primarily observational studies demonstrated modest benefits for disease free survival $(\mathrm{HR}=0.81,95 \%$ CI:0.72-0.91, $p=0.0007)$ but no effect on overall survival $(\mathrm{HR}=0.88$; 95\% CI:0.77-1.01, $p=0.07){ }^{32}$ Given the uncertain benefits, and concern for adverse side effects, our patient declined chemotherapy.

Repeat radiotherapy for this anal recurrence was another consideration. Particularly given the patient's profound response to neoadjuvant therapy during his first recurrence over two years prior. However, there is again a paucity of evidence to guide re-irradiation. The NCCN rectal cancer guidelines suggest re-irradiation should be delivered if it can be tolerated and is feasible, citing some small studies espousing the relative safety of this approach. ${ }^{28,33-35}$ One large retrospective series of resectable locally recurrent rectal cancers found that those who received repeat radiation as part of their treatment had longer overall survival, and increased time to local recurrence or metastasis compared to patients who were not re-irradiated. ${ }^{36}$ However, our patient had previously received a large cumulative dose of radiation after his first recurrence, and had significant side effects such that he would not consider re-irradiation a viable option.

Another situation we considered was whether this was a new primary neoplasm of the anal canal. Unlike squamous cell carcinoma (SCC), adenocarcinomas are only rarely encountered in the anus, accounting for less than $10 \%$ of anal cancers. ${ }^{37,38}$ Anal adenocarcinomas can be categorized into two types according to their origin: those which arise from the anal glands, usually in conjunction with a fistula; or "Colorectal-type" anal adenocarcinomas which are thought to arise from rectal glandular cells in the anal transition zone mucosa. ${ }^{37}$ Histologically, these latter lesions are identical to ordinary colorectal adenocarcinoma, and represent an interesting diagnostic and therapeutic dilemma, as they can be difficult to distinguish from low rectal cancers. This diagnosis can be particularly important, as anal adenocarcinomas have a worse prognosis than rectal cancer, as they more frequently metastasize to the inguinal and femoral nodal basins. ${ }^{37,38}$ In the presented case, histopathology could not differentiate between the original tumor and the tumor recurrence at the anal margin; although, this does not necessarily rule out the possibility of a new primary cancer. Analysis of the tumor genotype, and comparison between the index and the recurrent tumor theoretically could help determine whether they were related. However, this type of testing is not available locally. Fortunately, management options for localized anal adenocarcinoma, and those for localized rectal cancer recurrence at the anus are similar. ${ }^{38}$

Going forward, the question remains as to the cause of this exceedingly rare site of rectal cancer metastasis. Most of the similar case presentations in the literature occurred following some damage to the anal epithelial layer. However, in the current case the patient had no anal disease, and had no significant trauma from instrumentation of his anus. Previously minor trauma from the hooks of a Lone Star retractor or those of a Gelpi retractor have been implicated in tumor cell implantation in the anus. ${ }^{22,23}$ However, these instruments were never used in any of this patient's prior operations. The patient also had a significant stenosis at his original colorectal anastomosis, requiring multiple dilatations following his index operation 5 years prior. It remains a possibility that some minor perianal trauma that went unnoticed to the patient and the surgeon during one of these dilatation procedures or one of his past cancer operations led to the current presentation many years later. 


\section{Conclusion}

Isolated metachronous metastasis to the anus is an extremely rare site of recurrence for rectal adenocarcinoma, with only a small number of cases reported in the literature. Therapeutic options can be extrapolated from those performed for other locally recurrent rectal cancers or from isolated metachronous metastases to the liver. Regardless of the approach, patient-informed care is paramount. A firm understanding of the quality of the available evidence (or lack thereof) for conventional therapeutic options such as chemotherapy, radiation, and aggressive surgery in this setting is essential in order to help select the best therapeutic option.

\section{Abbreviations}

APR, abdominal perineal resection; CEA, carcinoembryonic antigen; CAPEOX, capecitabine plus oxaliplatin chemotherapy; FOLFOX, folinic acid plus 5-fluorouracil and oxaliplatin chemotherapy; NCCN, National Comprehensive Cancer Network; SCC, squamous cell carcinoma.

\section{Ethics Approval and Informed Consent for Publication}

Informed consent for publication of the patient's medical details and photographs was obtained from the patient prior to publication. Institutional approval was obtained from the University of Manitoba's Health Research Ethics Board. File number: HS25267 (H2021:410).

\section{Acknowledgments}

This work is original and is not under consideration elsewhere. This work has not been presented at any scientific meetings.

\section{Funding}

There was no specific funding for this research.

\section{Disclosure}

The authors report no conflicts of interest in this work.

\section{References}

1. World Health Organization (WHO). GLOBOCAN Database [Internet]; 2020 [cited October 2, 2021]. Available from: http://gco.iarc.fr/today/ home. Accessed December 24, 2021.

2. Siegel RL, Miller KD, Fuchs HE, Jemal A. Cancer statistics, 2021. CA Cancer J Clin. 2021;71(1):7-33.

3. Guiss RL. The implantation of cancer cells within a fistula in ano: case report. Surgery. 1954;36(1):136-139.
4. Gupta R, Kay M, Birch DW. Implantation metastasis from adenocarcinoma of the colon into a fistula-in-ano: a case report. Can J Surg. 2005;48(2):162-163.

5. Murata A, Takatsuka S, Shinkawa H, Kaizaki R, Hori T, Ikehara T. [A case report of metastatic anal fistula cancer treated with neoadjuvant chemotherapy]. Gan to Kagaku Ryoho. 2014;41(12):1869-1871. Japanese.

6. Gomes RM, Kumar RK, Desouza A, Saklani A. Implantation metastasis from adenocarcinoma of the sigmoid colon into a perianal fistula: a case report. Ann Gastroenterol. 2014;27 (3):276-279

7. Benjelloun EB, Aitalalim S, Chbani L, Mellouki I, Mazaz K, Aittaleb K. Rectosigmoid adenocarcinoma revealed by metastatic anal fistula. The visible part of the iceberg: a report of two cases with literature review. World J Surg Oncol. 2012;10(1):209.

8. Tomimaru Y, Ohue M, Noura S, et al. [A case of anal fistula cancer probably developing from intraluminal dissemination of rectal cancer]. Gan to Kagaku Ryoho. 2005;32(11):1776-1778. Japanese.

9. Rollinson PD, Dundas SA. Adenocarcinoma of sigmoid colon seeding into pre-existing fistula in ano. Br J Surg. 1984;71(9):664-665.

10. Kouraklis G, Glinavou A, Kouvaraki M, Raftopoulos J, Karatzas G. Anal lesion resulting from implantation of viable tumour cells in a pre-existing anal fistula. A case report. Acta Chir Belg. 2002;102 (3):212-213.

11. Ishiyama $\mathrm{S}$, Inoue $\mathrm{S}, \mathrm{Kobayashi} \mathrm{K}$, et al. Implantation of rectal cancer in an anal fistula: report of a case. Surg Today. 2006;36(8):747-749.

12. Wakatsuki K, Oeda Y, Isono T, et al. Adenocarcinoma of the rectosigmoid colon seeding into pre-existing anal fistula. Hepatogastroenterology. 2008;55(84):952-955.

13. Buzatti KC, de LR, Reis I, Daldegan I, Rodrigues B, Rectal cancer metastasis to an anal fissure. Clin Gastroenterol Treatment. 4(2). Available from:: https://clinmedjournals.org/articles/jcgt/journal-ofclinical-gastroenterology-and-treatment-jegt-4-061.php?jid=jcgt. Accessed December 24, 2021

14. Algallai M, Moftah M, Manita MA, Eldruki SI. Colorectal cancer implantation metastasis in haemorrhiodectomy wound, case report. Open J Gastroenterol. 2019;9(9):185-191.

15. Hsu T-C, Lu I-L. Implantation of adenocarcinoma on hemorrhoidectomy wound. Int J Colorectal Dis. 2007;22(11):1407-1408.

16. Abbasakoor F, Srivastava V, Swarnkar K, Stephenson BM. Implantation anal metastases after out-patient treatment of haemorrhoids. Ann R Coll Surg Engl. 2004;86(1):38-39.

17. Gujral DM, Bhattacharyya S, Hargreaves P, Middleton GW. Metastatic rectal adenocarcinoma within haemorrhoids: a case report. J Med Case Rep. 2008;2(1):128.

18. Liasis L, Papaconstantinou HT. Colorectal cancer implant in an external hemorrhoidal skin tag. Proc (Bayl Univ Med Cent). 2016;29(2):194-195.

19. Cantos-Pallarés M, García-Armengol J, Mulas-Fernández C, et al. Metástasis cutáneas perianales de adenocarcinoma colorrectal Revista Española de Enfermedades Digestivas. 2012;104(1):41-42.

20. Meshikhes A-WN. An unusual implantation of a rectosigmoid adenocarcinoma into a hemorrhoidectomy scar. Ann Saudi Med. 1998;18 (3):242-243.

21. De Friend DJ, Kramer E, Prescott R, Corson J, Gallagher P. Cutaneous perianal recurrence of cancer after anterior resection using the EEA stapling device. Ann R Coll Surg Engl. 1992;74 (2):142-143

22. Tranchart H, Benoist S, Penna C, Julie C, Rougier P, Nordlinger B. Cutaneous perianal recurrence on the site of Lone Star Retractor after J-pouch coloanal anastomosis for rectal cancer: report of two cases. Dis Colon Rectum. 2008;51(12):1850-1852.

23. Zinzindohoue F, Penna C, Parc R. Adenocarcinoma arising on the site of a Gelpi retractor after coloanal anastomosis for rectal cancer. $\mathrm{Br}$ J Surg. 1997;84(3):362. 
24. Sasaki S, Sugiyama M, Nakaji Y, et al. Anal metastasis of rectal cancer-adenocarcinoma of squamous cells: a case report and literature review. Surg Case Rep. 2017;21(3):55.

25. Pappou E. Chapter 31: proctectomy. In: Steele SR, Hull TL, Read TE, Saclarides TJ, Senagore AJ, Whitlow CB, eds. The ASCRS Textbook of Colon and Rectal Surgery. 3rd ed. Springer International Publishing; 2016:517-533.

26. Mirnezami AH, Sagar PM, Kavanagh D, Witherspoon P, Lee P, Winter D. Clinical algorithms for the surgical management of locally recurrent rectal cancer. Dis Colon Rectum. 2010;53(9):1248-1257.

27. Roeder F, Goetz J-M, Habl G, et al. Intraoperative Electron Radiation Therapy (IOERT) in the management of locally recurrent rectal cancer. BMC Cancer. 2012;11(12):592.

28. NCCN. National Comprehensive Cancer Network Clinical Practice Guidelines in Oncology (NCCN Guidelines): rectal Cancer (Version 2.2021); 2021 [cited 2021 Oct 2]. Available from: https://www.nccn. org/guidelines/guidelines-detail?category=1\&id=1461. Accessed December 24, 2021.

29. van den Brink M, Stiggelbout AM, van den Hout WB, et al. Clinical nature and prognosis of locally recurrent rectal cancer after total mesorectal excision with or without preoperative radiotherapy. J Clin Oncol. 2004;22(19):3958-3964.

30. Asoglu O, Karanlik H, Muslumanoglu M, et al. Prognostic and predictive factors after surgical treatment for locally recurrent rectal cancer: a single institute experience. Eur J Surg Oncol. 2007;33(10):1199-1206.

31. Harris CA, Solomon MJ, Heriot AG, et al. The outcomes and patterns of treatment failure after surgery for locally recurrent rectal cancer. Ann Surg. 2016;264(2):323-329.
32. Wang Z-M, Chen -Y-Y, Chen -F-F, Wang S-Y, Xiong B. Perioperative chemotherapy for patients with resectable colorectal hepatic metastasis: a meta-analysis. Eur J Surg Oncol. 2015;41 (9):1197-1203.

33. Das P, Delclos ME, Skibber JM, et al. Hyperfractionated accelerated radiotherapy for rectal cancer in patients with prior pelvic irradiation. Int J Radiat Oncol Biol Phys. 2010;77(1):60-65.

34. Guren MG, Undseth C, Rekstad BL, et al. Reirradiation of locally recurrent rectal cancer: a systematic review. Radiother Oncol. 2014;113(2):151-157.

35. Valentini V, Morganti AG, Gambacorta MA, et al. Preoperative hyperfractionated chemoradiation for locally recurrent rectal cancer in patients previously irradiated to the pelvis: a multicentric Phase II study. Int J Radiat Oncol Biol Phys. 2006;64(4):1129-1139.

36. Dresen RC, Gosens MJ, Martijn H, et al. Radical resection after IORT-containing multimodality treatment is the most important determinant for outcome in patients treated for locally recurrent rectal cancer. Ann Surg Oncol. 2008;15(7):1937-1947.

37. Lukovic J, Kim JJ, Krzyzanowska M, Chadi SA, Taniguchi CM, Hosni A. Anal adenocarcinoma: a rare malignancy in need of multidisciplinary management. JCO Oncol Pract. 2020;16(10):635-640.

38. Anwar S, Welbourn H, Hill J, Sebag-Montefiore D. Adenocarcinoma of the anal canal - a systematic review. Colorectal Dis. 2013;15 (12):1481-1488.
International Medical Case Reports Journal

\section{Publish your work in this journal}

The International Medical Case Reports Journal is an international, peer-reviewed open-access journal publishing original case reports from all medical specialties. Previously unpublished medical posters are also accepted relating to any area of clinical or preclinical science. Submissions should not normally exceed 2,000 words or 4

\section{Dovepress}

published pages including figures, diagrams and references. The manuscript management system is completely online and includes a very quick and fair peer-review system, which is all easy to use. Visit http://www.dovepress.com/testimonials.php to read real quotes from published authors. 